\title{
Altura del salto vertical en jugadores de balonmano (contribución del componente muscular, elástico, coordinativo $)^{1}$
}

https://doi.org/10.21830/9789585284814.01

\author{
Andrés Alonso Acevedo-Mindiola², Brian Johan Bustos Viviescas', \\ José Alexander Ortiz Novoa 4 , Rafael Enrique Lozano Zapata ${ }^{5}$, \\ Nelson Orlando Clavijo Gutiérrez', Carlos Alberto Hernández Camargo 7 , \\ Leidy Estefanía Rodríguez Acuña ${ }^{8}$
}

\section{Resumen}

Objetivo: describir la altura del salto vertical (SV) y la contribución del componente muscular (CM), elástico (CE) y coordinativo (CC), al igual que el índice elástico (IE) y utilización de los brazos en el SV (UB) de jugadores de balonmano (Selección

1 El texto del capítulo es el primer avance de una línea de investigación en desarrollo. La financiación de este trabajo ha sido propia.

2 Licenciado en Educación Básica con Énfasis en Educación Física, Recreación y Deportes de la Universidad de Pamplona (Sede Villa del Rosario). Contacto: andres.acevedo@unipamplona.edu.co. Orcid: https://orcid.org/0000-0003-0125-7265

3 Docente de la Fundacion Universitaria Juan de Castellanos. Maestría en Actividad Física y Entrenamiento Deportivo - Universidad Montrer (en formación). Especialista en Métodos y Técnicas de Investigación - Fundación Universitaria Claretiana. Observatorio de Investigación en Ciencias de la Actividad Física y el Deporte. Contacto: bjbustos@jdc.edu.co. Orcid: https://orcid.org/0000-0002-4720-9018

4 Licenciado en Educación Básica con Énfasis en Educación Física, Recreación y Deportes de la Universidad de Pamplona (Sede Villa del Rosario). Contacto: jose.novoa@unipamplona.edu.co. Orcid: https://orcid.org/0000-0003-1707-3719

5 Magíster en Educación Física mención en Fisiología del ejercicio. PhD. en Ciencias de la Actividad Física y el Deporte, docente de la Universidad de Pamplona (Sede Villa del Rosario). Contacto: rafaenloza@unipamplona.edu.co. Orcid: https://orcid.org/0000-0002-6239-5883

6 Magíster en Ciencias de la Actividad Física, PhD. en Educación, Rector de Institución Educativa La Garita. Contacto: nelsonclavijo@usantotomas.edu.co. Orcid: https://orcid.org/0000-0002-1755-4830

7 Esp. en Entrenamiento Deportivo - Universidad de Pamplona. Director de Rendimiento Deportivo del Cúcuta Deportivo S.A. Contacto: carloshernandezc@unipamplona.edu.co. Orcid: https://orcid. org/0000-0001-7174-4806

8 Estudiante de la Licenciatura en Educación Básica con Énfasis en Educación Física, Recreación y Deportes de la Universidad de Pamplona. Contacto: leidy.rodriguez3@unipamplona.edu.co. Orcid: https:// orcid.org/0000-0001-7684-112X 
Norte de Santander), participantes en el preclasificatorio a Juegos Nacionales del 2015 en Colombia. Metodología: este estudio descriptivo tuvo un enfoque cuantitativo con un diseño de campo y una muestra a conveniencia constituida por ocho deportistas que fueron evaluados en el salto desde media sentadilla ( $\mathrm{SJ}$ ), salto en contramovimiento con manos en la cintura $(\mathrm{CMJ})$ y salto Abalakov (ABK). Luego se determinó, por medio de ecuaciones, el porcentaje de contribución del CM, CE, CC, IE y UB en el SV. Para la recolección de datos se utilizó: báscula Tanita BC-730, tallímetro de pared Seca 206, computador portátil con sistema operativo Windows 7, software Axon Jump Versión 4.02 y plataforma de contacto AXON JUMP. Resultados: los datos obtenidos fueron tabulados y analizados en el programa Microsoft Excel Office 2013, se observó en SJ 31,76+4,1 cm, en CMJ $34,51+5,07 \mathrm{~cm}$ y por último en $\mathrm{ABK} 41,41+5,46 \mathrm{~cm}$. Se encontró una mayor contribución del componente muscular $(76,7+3,82 \%)$ en comparación al CE $(6,64+1,92 \%)$ y CC $(16,66+4,37 \%)$. En cuanto a la UB $(19,99+6,41 \%)$ en el salto vertical fue mayor que el IE $(8,66+2,57 \%)$. Conclusión: en la ejecución del salto hay una escasa participación o contribución del componente elástico que afecta el rendimiento del salto vertical de los deportistas estudiados.

Palabras clave: balonmano; deporte; fuerza muscular; rendimiento atlético; salto vertical.

\section{Introducción}

En la actualidad el deporte de élite es cada vez más exigente y compeł titivo, pues demanda del deportista alta preparación física con el propósito de que se encuentre en la mejor forma (Barraza, Yáneez, Tuesta, Núnez, Zamora \& Rosales, 2015). Esto sucede con el balonmano, que exige a sus atletas mucha preparación para alcanzar un óptimo nivel de rendimiento en la competencia. Esta disciplina se caracteriza por ser un deporte acíclico, puesto que implica gran desarrollo de la resistencia aeróbica, así mismo, se evidencia una gran predominancia del metabolismo anaeróbico aláctico (Barraza et al., 2015) debido a los diferentes movimientos realizados durante el juego, donde intervienen las capacidades de fuerza, potencia, velocidad, resistencia y flexibilidad (Jiménez, 2015), indispensables para ejecutar con eficacia los fundamentos técnicos individuales como lanzamientos, saltos y cambios de dirección, fundamentales en este deporte (Marques, Van Den Tillaar, Vescovi \& González-Badillo, 2007; Aguilar-Martínez, Chirosa, Martín, Chirosa, \& Cuadrado-Reyes, 2012). 
Con referencia a lo anterior, tener una alta potencia muscular es fundamental en la mayoría de las disciplinas deportivas (Quiroga, Bustamante, Avendaño, Cáceres \& Urrea, 2016), dado que es un factor importante en el rendimiento de los jugadores dentro del campo de juego (Barraza et al., 2015). En el caso del balonmano, esta capacidad está determinada por su ejercicio más representativo: "el salto", en donde el atleta que practica y compite debe entrenar este movimiento para tener un óptimo desempeño y para mejorar su potencia, la cual está determinada por la altura del salto (Quiroga et al., 2016). Dentro de los diferentes patrones de movimientos que intervienen en este deporte, el salto es una habilidad motriz fundamental, ya que algunos gestos técnicos como el lanzamiento en suspensión o el pase en suspensión, entre otras jugadas individuales o colectivas, implican la ejecución de un salto en diferentes situaciones de competición, tanto en acciones de ataque como en defensa (Garrido, González, Expósito, Sirvent \& García, 2012).

Los saltos verticales pueden usarse como una evaluación primordial del deportista para estudiar su capacidad generadora de fuerza explosiva y fuerza elástica en extremidades inferiores (Pääsuke, Ereline \& Gapeyevas, 2013), pues son estas las cualidades físicas que se ven implicadas durante el salto vertical (Mariño, Becerra \& Bugallo, 2012). Además, el salto es un gesto que constituye un indicador de la capacidad de generar potencia en un movimiento explosivo (Serrato, 2008). En la fuerza elástica predomina la contribución del componente elástico que actúa por efecto del estiramiento previo, lo que sirve para almacenar cierta cantidad de energía elástica que puede ser utilizada en la fase concéntrica (Serrato, 2008), por lo cual es menor la capacidad contráctil y los mecanismos nerviosos de reclutamiento (Mariño, Becerra \& Bugallo, 2012). También, por ser un gesto complejo, se requiere del aporte del componente contráctil y coordinativo (Serrato, 2008).

En consecuencia, es fundamental y necesario realizar la evaluación de la altura del salto vertical por medio de diferentes tipos de pruebas, con el fin de mejorar los niveles de fuerza explosiva y fuerza elástica (Serrato, 2008), así como de identificar futuros talentos deportivos que se encuen- 
tren dentro de las características establecidos en la literatura científica (Bosco, 1994; Barraza et al., 2015). De igual forma, los datos obtenidos en las pruebas de salto permitirán al entrenador realizar una planificación del entrenamiento adecuada a la capacidad física de los deportistas (Gutiérrez, 2010), de esta manera se llevan a cabo correctamente tanto un seguimiento como un control de las cargas de trabajo en la preparación física, lo que se logra a través de los ajustes y modificaciones del volumen, intensidad y densidad (Sánchez-Alcaraz, Pérez \& Pérez, 2013).

En ese contexto, el presente estudio pretende describir la altura del salto SJ, CMJ y $\mathrm{ABK}$, pues son escasos los estudios científicos que existen, tanto a nivel regional como nacional, acerca de los jugadores de balonmano. Por tanto, existe la necesidad de poder comparar los resultados de los deportistas pertenecientes a la de la liga de balonmano del departamento Norte de Santander, frente a deportistas de alto rendimiento de otras ciudades y países. De esta manera, se establecerán las principales características de los deportistas que participaron en el preclasificatorio a los XX Juegos Nacionales 2015; información que podría ayudar a encaminar mejor la preparación física y realizar las modificaciones pertinentes en la periodización del entrenamiento para lograr una mejor performance.

Por ello, este estudio tiene como objetivo describir la altura del salto vertical y la contribución de cada uno de sus componentes, al igual que determinar el índice elástico y dar cuenta de la utilización de los brazos por parte de los jugadores de balonmano de la Selección Norte de Santander, dado que es un parámetro fundamental para la planificación y el control de las cargas de entrenamiento, lo que contribuye a optimizar el rendimiento deportivo.

\section{Metodología}

\section{a. Diseńo del estudio}

El estudio desarrolló una investigación descriptiva de enfoque cuantitativo y un diseño de campo que consistió en la recolección de datos directamente de los sujetos investigados, de este modo se obtuvieron datos primarios de la realidad donde ocurren los hechos, sin manipulación o control de variable 
alguna (Arias, 2012). Igualmente, se utilizó el criterio de muestra a conveniencia, puesto que la selección se realizó gracias al acceso y proximidad de los sujetos para los investigadores (Scribano, 2007).

\section{b. Participantes}

Los participantes de este estudio fueron ocho hombres categoría abierta (edad 17,9 $\pm 1,89$ años, talla $170,0 \pm 0,04 \mathrm{~cm}$, peso corporal $60,55 \pm 9,65 \mathrm{~kg}$ ) que se encontraban en etapa precompetitiva realizando una frecuencia de 4 sesiones semanales. Entre los criterios de inclusión necesarios para participar en el estudio se debía pertenecer a la liga de balonmano de Norte de Santander, tener regularidad en la asistencia a los entrenamientos y no haber consumido licor el día antes de la prueba. Por otra parte, algunos de los criterios para la exclusión del estudio fueron: presentar alguna lesión o patología osteomuscular, metabólica o cardiorrespiratoria, así como el consumo de algún medicamento que impidiera la realización del estudio.

Cabe agregar que esta investigación se realizó teniendo en cuenta los estándares éticos establecidos para investigaciones en ciencias del deporte y del ejercicio (Harriss \& Atkinson, 2013), dado que todos los participantes del estudio fueron informados acerca de los objetivos de este, así como de los procedimientos a llevar a cabo durante su desarrollo, además, firmaron un consentimiento por escrito. De igual manera, por tratarse de menores de edad, los padres de familia fueron informados con respecto a los detalles del estudio y firmaron también el consentimiento para autorizar la participación de sus hijos.

\section{c. Métodos y procedimientos}

Las pruebas realizadas se basan en el test de Bosco (1994), se usó una plataforma de contacto que permite la evaluación y caracterización de los parámetros funcionales del salto en cada uno de los jugadores evaluados y la medición de la fuerza dinámica de las extremidades inferiores. Igualmente, se realizó la prueba de laboratorio conocida como el test de salto sobre plataforma de contacto, que permite estudiar los diferentes componentes que intervienen en el salto vertical como son: 
- Componente coordinativo

- Componente muscular

- Componente elástico

Para evaluar estos componentes se realizaron en un mismo día los siguientes saltos:

- Squat Jump o salto desde media sentadilla con manos en la cintura (involucra solamente el componente muscular).

- CMJ o salto en contramovimiento con manos en la cintura (involucra el componente muscular y el componente elástico).

- Abalakov o salto con braceo (involucra los tres anteriores componentes).

En cada tipo de salto el deportista realizó 2 intentos, así, para el estudio, se tomó el mejor resultado. Luego, con la altura $(\mathrm{cm})$ de los saltos se determinaron los porcentajes en que cada componente contribuye a la altura del salto vertical, así como los porcentajes de contribución del componente elástico y de coordinación de los brazos. Cabe agregar que antes de empezar las pruebas de salto todos los deportistas realizaron un calentamiento de 10 minutos, luego se continuó con la explicación de la técnica de ejecución de los distintos saltos. El descanso fue de tres minutos entre cada salto para evitar posibles interferencias debidas al cansancio (Ferragut, Cortadellas, Arteaga \& Calbet, 2003; Vila, Manchado, Rodríguez, Abraldes, Alcaraz \& Ferragut, 2012).

Para determinar el porcentaje en que cada componente contribuye a la altura del salto vertical se utilizaron las siguientes ecuaciones (Alba, 2005):

$\%$ contribución del componente muscular = Altura en SJ / altura en Abalakov x 100 .

\% contribución componente elástico = (Altura en CMJ - Altura en SJ $) /$ Altura en Abalakov x 100.

$\%$ contribución componente coordinativo $=100-(\%$ contribución del componente muscular $+\%$ contribución componente elástico).

La diferencia porcentual en la altura lograda entre el salto desde media sentadilla y el salto en contramovimiento se define como índice de elasticidad 
o porcentaje de utilización del componente elástico (Alba, 2005), en donde CMJ es el resultado del salto en contramovimiento $(\mathrm{cm})$ y SJ es el resultado del salto desde media sentadilla $(\mathrm{cm})$.

$$
\text { Ind. Elasticidad }(\%)=(C M J-S J) / S J \times 100
$$

Este índice debe encontrarse entre 10 y 30\%. Por debajo de 10\% se considera buen desarrollo del componente muscular con relación al componente elástico, recomendándose entrenamiento pliométrico. Por encima de 30\% es bueno el desarrollo del componente elástico en relación con el componente muscular, recomendándose entrenar la fuerza máxima (Alba, 2005).

Por último, para determinar el porcentaje de utilización o contribución de los brazos, se utilizó la siguiente fórmula (Alba, 2005), en la cual ABK es el salto vertical con braceo $(\mathrm{cm})$ y CMJ es el resultado del salto en contramovimiento $(\mathrm{cm})$.

$$
\% \text { utilización de Brazos }=(\mathrm{ABK}-\mathrm{CMJ}) / \mathrm{CMJ} \text { x } 100
$$

\section{d. Instrumentos}

Para la recolección de datos se utilizaron los siguientes instrumentos:

- Báscula Tanita BC-730: utilizada para obtener el peso corporal de cada deportista, precisión de $100 \mathrm{~g}$.

- Tallímetro de pared Seca 206: empleado para medir la estatura $(0-220 \mathrm{~cm})$, precisión $1 \mathrm{~mm}$.

- Computador portátil con sistema operativo Windows 7: utilizado para establecer a través del software los saltos a medir en la plataforma de contacto.

- Software Axon Jump Versión 4.02: empleado para utilizar la plataforma de contacto.

- Plataforma de contacto Axon Jump: utilizada para medir la altura de los saltos.

\section{e. Plan de análisis estadístico}

Para la tabulación y el análisis de los resultados obtenidos se utilizó el programa Microsoft Excel Office 2013, en donde se calculó la estadística 
descriptiva de media y \pm desviación estándar (DE) de todos los saltos evaluados, así como la contribución de cada componente, el índice de elasticidad y la utilización de los brazos.

\section{Resultados}

A continuación, se exponen detalladamente los resultados obtenidos en el presente estudio. En en la Tabla 1 se presentan los resultados con respecto a la altura en los saltos SJ, CMJ y ABK realizados por los jugadores de balonmano que participaron en esta investigación. En el salto SJ los deportistas

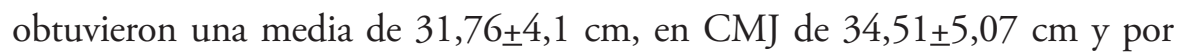
último en $\mathrm{ABK} 41,41 \pm 5,46 \mathrm{~cm}$.

Tabla 1. Altura del salto vertical SJ, CMJ y ABK

\begin{tabular}{lccc}
\hline \multicolumn{1}{c}{ Salto } & SJ (cm) & CMJ (cm) & ABK (cm) \\
\hline Media & 31,76 & 34,51 & 41,41 \\
DE & 4,10 & 5,07 & 5,46 \\
\hline
\end{tabular}

Leyenda: DE - Desviación Estándar

Fuente: elaboración propia.

La Tabla 2 muestra los resultados del porcentaje de contribución de cada componente en el salto vertical. Aquí se evidencian diferencias, dado que el porcentaje de contribución del componente muscular fue mayor con respecto a los otros dos componentes, con un $76,7 \pm 3,82 \%$; por otro lado, el porcentaje de contribución del componente elástico fue menor, con un $6,64 \pm 1,92 \%$, y, finalmente, la contribución del componente coordinativo fue mayor que el componente elástico y menor que el componente muscular, con un $16,66 \pm 4,37 \%$. 
Tabla 2. Porcentaje (\%) de contribución del componente muscular, elástico y coordinativo en el salto vertical

\begin{tabular}{llll}
\hline \% Contribución & $\begin{array}{c}\text { Componente } \\
\text { Muscular }\end{array}$ & $\begin{array}{c}\text { Componente } \\
\text { Elástico }\end{array}$ & $\begin{array}{l}\text { Componente } \\
\text { Coordinativo }\end{array}$ \\
\hline Media & 76,70 & 6,64 & 16,66 \\
DE & 3,82 & 1,92 & 4,37 \\
\hline
\end{tabular}

Leyenda: DE - Desviación Estándar

Fuente: elaboración propia.

Para terminar, en la Tabla 3 se presenta el porcentaje de utilización del componente elástico o índice de elasticidad y el porcentaje de utilización o contribución de los brazos en el salto vertical, en donde el porcentaje de índice de elasticidad fue de $8,66 \pm 2,57 \%$, lo que indica un buen desarrollo del componente muscular en relación con el componente elástico, por otro lado, el porcentaje de utilización de brazos fue de $19,99 \pm 6,41 \%$.

Tabla 3. Índice de elasticidad y utilización de brazos en el salto vertical ejecutado por los deportistas de este estudio

\begin{tabular}{lcc}
\hline Porcentaje (\%) & Índice de elasticidad & Utilización de brazos \\
\hline Media & 8,66 & 19,99 \\
DE & 2,57 & 6,41 \\
\hline
\end{tabular}

Leyenda: DE - Desviación Estándar

Fuente: elaboración propia.

\section{Discusión}

Este análisis tuvo por objetivo describir la altura del salto vertical y la contribución de cada uno de sus componentes, así mismo, buscó determinar el índice elástico y dar cuenta de la utilización de los brazos en el salto vertical de jugadores de balonmano de la Selección Norte de Santander. Teniendo en cuenta los resultados obtenidos, en la altura de los saltos se pudo evidenciar que existen diferencias en el rendimiento para cada uno de los ejercicios evaluados, 
en forma grupal, con los jugadores de balonmano de esta investigación. Así, se hallaron diferencias entre el desarrollo de los saltos analizados SJ y CMJ, tal como se muestra en la Tabla 1; por ejemplo, se observa que el promedio del salto CMJ supera en $2,75 \mathrm{~cm}$ al salto SJ. También fueron contrastados los saltos $\mathrm{ABK}$ contra el rendimiento en $\mathrm{CMJ}$, en el cual se encontró un valor superior del salto $A B K$, como se puede observar en la Tabla 1, dado que el promedio obtenido supera en $6,9 \mathrm{~cm}$ al salto CMJ.

De manera semejante, se observan diferencias al comparar los resultados de este estudio con los encontrados en otras investigaciones con jugadores de balonmano masculino. Por ejemplo, los valores obtenidos en el estudio de Massuçaa y Fragoso (2013) —quienes estudiaron 34 jugadores profesionales de balonmano adultos (edad 23,4 $\pm 4,7$ años), 18 de los cuales fueron clasificados como exitosos (edad 23,0 $\pm 3,8$ ańos) y tuvieron una altura en el salto SJ de $34 \pm 6 \mathrm{~cm}$, en CMJ $36 \pm 6 \mathrm{~cm}$, en ABK $43 \pm 6 \mathrm{~cm}$, y 16 como menos exitosos (edad, $23,8 \pm 5,5$ años) con una altura en el salto $S J$ de $37 \pm 6 \mathrm{~cm}$, en CMJ $39 \pm 6 \mathrm{~cm}$, en $\mathrm{ABK} 45 \pm 7 \mathrm{~cm}$ - son superiores a los resultados de este estudio. De igual forma, en un estudio realizado por Garrido et al. (2012) con 50 deportistas de alto nivel pertenecientes al balonmano masculino, se obtuvo una altura superior a la conseguida por los deportistas que participaron en nuestra investigación, con un promedio en el salto $S J$ de $33,13+4,53 \mathrm{~cm}$, en CMJ 36,42+4,40 cm y en ABK de 44,27+4,39 cm. Esto nos indica un bajo rendimiento en el salto y poco fortalecimiento en miembros inferiores de los jugadores de balonmano estudiados, lo que se reflejará en competiciones, en donde la potencia y fuerza explosiva son factores determinantes para triunfar en este deporte (Salazar, 2009).

Por otro lado, en la investigación realizada por Sebastia-Amat, EspinaAgullo y Chinchilla-Mira (2017), 26 jugadores de género masculino que ocupaban la posición de portero dentro de sus respectivos equipos de la comunidad Valenciana fueron divididos en tres grupos según su edad, teniendo en cuenta para la comparación el grupo 3, debido a que se encontraban en una edad comprendida entre 17 y 20 años, al igual que los deportistas estudiados en nuestro caso. Los resultados encontrados fueron: en SJ 28,99+6,03 cm, CMJ 32,12+6,93 cm y Abalakov 37,58+5,85 cm, valores inferiores a los obtenidos en el presente estudio, puesto que las demandas energéticas del portero 
en comparación con el resto de las posiciones que ocupan los jugadores no son tan exigentes (Srhoj, Marinovic \& Rogulj, 2002). Además, solo deben actuar en espacios reducidos realizando acciones explosivas en una fracción de segundos (Milanese, Piscitelli, Lampis \& Zancanaro, 2011) a diferencia del resto de jugadores que actúan en todo el terreno de juego.

De acuerdo a la contribución del componente muscular, coordinativo y elástico, son escasos los estudios que se encuentran en la literatura científica que determinen las variables que intervienen en el salto, por tal razón, se recomienda que en futuras investigaciones se tenga en cuenta la contribución de cada componente para encaminar, de una manera óptima, el entrenamiento deportivo enfocado en las debilidades o falencias, ya sea para lograr un buen desarrollo del componente muscular o del componente elástico. Además de lo anterior, y con el fin de mejorar la fuerza elástica, la inclusión de ejercicios pliométricos en la planificación del entrenamiento de balonmano con el establecimiento de objetivos a corto y largo plazo permitirá conseguir el máximo rendimiento en el desarrollo del juego real de esta disciplina. Los efectos de ejercicios pliométricos en la fuerza elástica han sido revisados por muchos autores que han comprobado, a través de diversas investigaciones, el aumento en el salto vertical con el entrenamiento pliométrico (Markovic, 2007). De igual manera, el entrenamiento pliométrico ha sido recomendado para deportes que requieren acciones explosivas y mejoras en la altura del salto vertical (Arenas, 2009).

En cuanto a la contribución de los brazos y el índice de elasticidad, también se observan diferencias muy marcadas, lo que ha permitido establecer que el perfil de fuerza elástica en jugadores de balonmano es, en general, bajo. Esta fuerza se estima por la diferencia porcentual entre ambos saltos, SJ y CMJ. En cambio, en la utilización de brazos se observa, como muestra la Tabla 3, un valor superior, lo que indica que hubo una mayor contribución de los brazos con respecto al componente elástico. Así mismo, el comportamiento de los deportistas durante la ejecución del salto con contramovimiento (CMJ) es superior con respecto al Squat Jump (SJ). Por ello, en la planificación de deportistas practicantes de balonmano con el fin de mejorar el rendimiento del salto vertical, es necesario incluir entrenamiento pliométrico para contribuir con la evolución y ganancia de saltabilidad, la cual se evidencia a través de la fuerza elástica. 
El balonmano colombiano es un deporte joven que consiguió ser incluido por primera vez como deporte oficial para los Juegos Nacionales de 2015 en Colombia; en esa ocasión la Federación Colombiana de Balonmano, creada en 2010, programó dos paradas clasificatorias a las justas nacionales. En la rama masculina participó la liga de Balonmano de Norte de Santander, para un total de 10 equipos participantes. Se presentaron las ligas de Antioquia, Quindío, Valle, San Andrés, Chocó, Cundinamarca, Boyacá, Risaralda, Cauca, Bogotá y la liga Norte. Antioquia y Valle dominaron el evento y disputaron las finales de los Juegos Nacionales; cabe anotar que estas son las ligas con más recorrido en nuestro país. Los resultados en el preclasificatorio a los XX Juegos Nacionales, organizados por el Departamento Administrativo del Deporte, la Recreación, la Actividad Física y el Aprovechamiento del Tiempo Libre (Coldeportes, 2015), no fueron representativos en triunfos para los deportistas de este estudio, pues la liga de balonmano de Norte de Santander no clasificó, lo que puede estar relacionado con el bajo rendimiento en el salto. El balonmano colombiano sigue creciendo, por ello, se espera que con este estudio se realicen las modificaciones pertinentes para conseguir un alto rendimiento deportivo en los próximos juegos del 2019.

\section{Conclusión}

Los deportistas evaluados presentan un bajo rendimiento en el salto vertical comparado con los valores arrojados por otros estudios. Sucede lo mismo con sus diferentes componentes e índices del salto, por lo que esta deficiencia en el componente del salto vertical puede afectar el desempeño durante las competencias.

\section{Referencias}

Aguilar, D., Chirosa, L., Martín, I., Chirosa, I., \& Cuadrado, J. (2012). Efecto del entrenamiento de la potencia sobre la velocidad de lanzamiento en balonmano. Revista Internacional de Medicina y Ciencias de la Actividad Física y del Deporte, 12(48), 729-744. http://cdeporte. rediris.es/revista/revista48/artefecto323.htm 
Alba, A. (2005). Test funcionales, cineantropometría y prescripción del entrenamiento en el deporte y la actividad fisica. Editorial Kinesis.

Arenas, J. (2009). Influencia de un plan de entrenamiento pliométrico de moderada intensidad en miembros inferiores sobre el indice elástico de las jugadoras de voleibol femenino de la institución educativa INEM José Félix de Restrepo con edades que oscilan entre los 14 y 17 años [tesis de pregrado]. Universidad de Antioquia (Medellín, Colombia).

Arias, F. (2012). El proyecto de investigación. Introducción a la metodología científica. Editorial Episteme.

Barraza, F., Yáñez, R., Tuesta, M., Núńez, P., Zamora, Y., \& Rosales, G. (2015). Perfil antropométrico por posición de juego en handbolistas chilenos. International Journal of Morphology, 33(3), 1093-1101. http://dx.doi.org/10.4067/S0717-95022015000300045

Bosco, C. (1994). La valoración de la fuerza con el test de Bosco. Paidotribo.

Departamento Administrativo del Deporte, la Recreación, la Actividad Física y el Aprovechamiento del Tiempo Libre. (2015). XX juegos deportivos nacionales "Carlos Lleras Restrepo"-2015. Acuerdo mediante el cual se fijan las bases de competencia para el campeonato de balonmano masculino y femenino [reglamento]. https://www.juegosnacionales.gov.co/ uploads/item/Balonmano.pdf

Ferragut, C., Cortadellas, J., Arteaga, R., \& Calbet, J. (2003). Predicción de la altura de salto vertical, importancia del impulso mecánico de la masa muscular de las extremidades inferiores. European Journal of Human Movement, 10, 7-22. http://dialnet.unirioja.es/servlet/ oaiart? codigo $=2279024$

Garrido, R., González, M., Expósito, I., Sirvent, J., \& García, M. (2012). Valores del Test de Bosco en función del deporte. PubliCE Standard. https://journal.onlineeducation.center/ api-oas/v1/articles/sa-T57cfb2715112d/export-pdf

Gutiérrez, A. (2010). La utilización del parámetro temporal en la actividad físico-deportiva. Acción Motriz, (4), 25-31. http://mdc.ulpgc.es/cdm/ref/collection/amotriz/id/67

Harriss, D., \& Atkinson, G. (2013). Ethical standards in sport and exercise science research: 2014 update. International Journal of Sports Medicine, 34(12), 1025-1028. http://dx.doi. org/10.1055/s-0033-1358756

Jiménez, A. (2015). Análisis comparativo de las caracteristicas cineantropométricas y de la condición física en jugadores de balonmano en edad juvenil [tesis de maestría inédita]. Universidad de León.

Mariń, N., Becerra, H., \& Bugallo, E. (2012). Análisis del rendimiento en el salto vertical de un grupo de deportistas del fútbol profesional colombiano. Revista actividad física y desarrollo humano, 4 (1), 24-31. http://revistas.unipamplona.edu.co/ojs_viceinves/index.php/ AFDH/article/view/328

Markovic, G. (2007). Does plyometric training improve vertical jump height? A meta-analytical review. British Journal of Sports Medicine, 41(6), 349-355. http://dx.doi.org/10.1136/ bjsm.2007.035113

Marqués, M., Van Den Tillaar, R., Vescovi, J., \& González, J. (2007). Relationship between throwing velocity, muscle power, and bar velocity during bench press in elite handball 
players. International Journal of Sports Physiology and Performance, 2(4), 414-422. https://www.ncbi.nlm.nih.gov/pubmed/19171959

Massuçaa, L., \& Fragoso, I. (2013). Enfoque multidisciplinar sobre el éxito en el balonmano. Apunts Medicina L'Esport, 48 (180), 143-151. https://www.apunts.org/ en-pdf-X0213371713597169

Milanese, C., Piscitelli, F., Lampis, C., \& Zancanaro, C. (2011). Anthropometry and body composit tion of female handball players according to competitive level or the playing position. Journal of Sports Sciences, 29(12), 1301-1309. https://doi.org/10.1080/02640414.2011.591419

Pääsuke, M., Ereline, J., \& Gapeyevas, H. (2013). La fuerza del músculo extensor de la rodilla y las características de la ejecución del salto vertical en chicos pre- y post-púberes. Revista de Entrenamiento Deportivo, 27(1), 1-9. https://journal.onlineeducation.center/api-oas/v1/ articles/sa-V57cfb2722f279/export-pdf

Quiroga, P., Bustamante, A., Avendaño, C., Cáceres, S., \& Urrea, S. (2016). Aumento de altura en salto en jugadores universitarios de voleibol. Apunts: Educación Física y Deportes, 4(126), 64-71. http://dx.doi.org/10.5672/apunts.2014-0983.es.(2016/4).126.07

Salazar, J. (2009). Evaluación de la fuerza explosiva en jugadoras de balonmano y voleibol. Revista Mexicana de Investigación en Cultura Fisica y Deporte, 1(1), 266-287. http://revista. ened.edu. $\mathrm{mx} /$ index.php/revistaconade/article/view/22

Sánchez, B., Pérez, D., \& Pérez, M. (2013). Fundamentos de la condición física en el pádel. Diego Marín Librero Editor.

Scribano, A. (2007). El proceso de investigación social cualitativo. Prometeo Libros.

Sebastia-Amat, S., Espina, J., \& Chinchilla, J. (2017). Perfil de salto vertical, velocidad, flexibilidad y composición corporal de porteros de balonmano en categorías inferiores. Retos, 32, 248-251. https://recyt.fecyt.es/index.php/retos/article/viewFile/56075/33853

Serrato, R. (2008). Medicina del deporte. Editorial Universidad del Rosario.

Srhoj, V., Marinovic, M., \& Rogulj, N. (2002). Position specific morphological characteristics of top-level male handball players. Collegium Antropologicum, 26(1), 219-227.

Vila, H., Manchado, C., Rodriguez, N., Abraldes, J., Alcaraz, P., \& Ferragut, C. (2012). Anthropometric profile, vertical jump, and throwing velocity in elite female handball players by playing positions. The Journal of Strength and Conditioning Research, 26(8), 2146-2155. https://doi.org/10.1519/JSC.0b013e31823b0a46 